\title{
Yoga-Based Cardiac Rehabilitation: Current Perspectives from Randomized Controlled Trials in Coronary Artery Disease
}

\section{Charo Bruce \\ Vinod Achan \\ Sudhir Rathore}

Department of Cardiology, Frimley Park Hospital, Frimley, Surrey, UK
Correspondence: Sudhir Rathore Department of Cardiology, Frimley Park Hospital, Frimley, Surrey GUI6 7UJ, UK Tel +4403006145000

Email Sudhir.rathore@nhs.net

\begin{abstract}
Coronary artery disease carries a high morbidity and mortality worldwide, and exercise-based cardiac rehabilitation programmes play a large role in secondary prevention. Exercise-based rehabilitation programmes are expensive, and in certain subgroups uptake is poor. Yoga has been suggested to show improvements in cardiovascular health which would support its use in cardiac rehabilitation programmes. We carried out a review of current randomized controlled trials to determine if yoga-based cardiac rehabilitation leads to reduced cardiac risk factors, and improved physiological and psychological outcomes in patients with coronary artery disease compared to standard care. Six randomized controlled studies were identified after a medical database search, and meta-analysis was carried out for the different outcomes. Overall, the addition of yoga to standard care resulted in improved subjective feeling of cardiac health and quality of life. There was also a trend towards improvement in left ventricular systolic function. Improvement in cardiac risk factors, MACE and psychological health in this cohort has still to be proven, but was not inferior to standard or enhanced care, and the benefits became more pronounced at longer follow-up. Future studies with longer follow-up and larger patient numbers would aid in accurately assessing the long-term benefit of yoga-based rehabilitation.
\end{abstract}

Keywords: yoga, cardiac rehabilitation, coronary artery disease

\section{Introduction}

Cardiovascular disease is the number one cause of death globally, accountable for an estimated $31 \%$ of annual deaths. ${ }^{1}$ Coronary artery disease is the most common form of heart disease with an estimated global prevalence of 126.5 million and an incidence of 10.6 million; in the UK it was primarily responsible for 311,519 hospital admissions and 64,132 deaths in $2018 .^{2}$

Following a coronary event, patients are more likely to have further events; while interventional and pharmacological therapies are often necessary, risk factor modification plays a crucial role in secondary prevention. Exercise-based cardiac rehabilitation (CR) is an established evidence-based programme that has been shown to reduce cardiovascular mortality and hospitalization, and improve physiological and psychological well-being in patients with cardiovascular disease. ${ }^{3}$ In CR physical activity is not undertaken in isolation, but as part of a comprehensive recovery programme including healthy eating, smoking cessation, medication adherence and stress management. Aerobic exercise reduces cardiovascular risk factors such as sedentary lifestyle, obesity and hypertension as well as promoting 
myocardial perfusion and decreasing myocardial oxygen demand. A total of 111 countries around the world offer exercise-base cardiac rehabilitation for patients with ischemic heart disease, with its use being a class 1 recommendation in both European and American guidelines. ${ }^{4-6}$ Traditionally aerobic exercise is used for CR; however, different forms of exercise have been trialled to improve CR uptake and enable tele-based CR and look at lowercost programmes.

Yoga combines physical poses with breath control and meditation as a form of exercise and weight loss programme. It has been successfully used in other rehabilitation programmes, such as adjunctive therapy in pulmonary rehab programmes for COPD and heart failure where it was shown to improve peak oxygen consumption and quality of life. ${ }^{7,8}$ It has also been shown to be of benefit in multiple other conditions such as anxiety and depression and chronic pain, and has even been suggested as a preventative strategy for COVID-19. ${ }^{9-11}$ The majority of studies looking at yoga-rehab in cardiovascular disease have been carried out in India, where the practice is more prevalent, and a low-cost model would improve availability and the familiarity is hoped to promote uptake. In countries with higher income and more developed rehabilitation programmes it was felt it may encourage participation in certain subgroups such as women, the elderly and those with low income who traditionally have poor uptake of conventional CR.

The randomized controlled trial data available at present have not produced a unified conclusion on the benefits of yoga-based rehabilitation; this is in part due to the heterogeneous nature of the studies, examining different patient groups, comparators and outcomes. This lack of consistency makes it difficult to determine its use in clinical practice. We carried out a review of current randomized controlled trials to discuss the evidence of yogabased cardiac rehabilitation and to consider if it improves physiological and psychological outcomes in patients following acute coronary disease compared to standard care.

\section{Methods}

\section{Search Strategy}

Studies were identified from searching Cochrane online Library, PubMed, Medline, EMCARE and CINAHL databases from inception to 23/08/2021 using search terms for "yoga" and "cardiovascular disease" or "coronary artery disease". The initial searches were supplemented by hand- searching the reference and citation lists of identified studies. Published studies from all years were included.

\section{Eligibility Criteria}

Studies were included if they looked at impact on cardiovascular outcomes of yoga-based cardiac rehabilitation compared to standard care for patients with acute coronary syndrome (ACS), following percutaneous coronary intervention or coronary artery bypass (CABG), or stable angina pectoris. Comparator could be standard care for that institution, which may only be health education advice, or a comprehensive cardiac rehabilitation programme. A minimum follow-up of 3 months was required. Only randomized controlled trials were included. Studies published in languages other than English were excluded.

\section{Outcomes}

Studies were included if they looked at the effect of the intervention on cardiovascular risk factors, physiological or psychological outcomes. This included: heart rate variability, blood pressure, body weight, lipid profile, glucose levels, echocardiography and self-reported measures of cardiac function, QOL, or anxiety or depression-focused questionnaires.

\section{Analysis}

Narrative and statistical analysis was carried out. Metaanalysis was carried out using Review Manager (RevMan) [Computer program], Version 5.4.1, The Cochrane Collaboration, 2020. Parameters from the studies were compared using inverse variance (IV) with a fixed effect model. The effect measure was analysed using standard mean difference to allow for different methods of measurement with $95 \%$ confidence intervals. Study correspondents were contacted for raw data if these were not available, and the studies were included if correspondents returned the relevant data.

\section{Results}

Our search yielded 571 unique studies; after review of title and abstract 13 studies were identified to meet eligibility criteria.

We identified 13 published studies that assessed outcomes in patients undergoing the addition of a yoga-based CR compared to conventional rehabilitation programmes. A total of 558 were excluded after screening of abstracts and titles. Following full analysis, a further 7 were excluded, and 6 were included in the review as seen in 
Figure 1. No studies were excluded for low quality. An overview of the 6 studies can be seen in Table 1 .

The trials are heterogeneous; they vary in size, patient population, primary outcomes and duration of follow-up. The trial with the longest follow-up and largest numbers (Prabhakaran et al) studied MACE and patient behaviour following acute MI but did not look at risk factors and LVEF. $^{12}$ One study only looked at patients post-CABG (Raghuram et al), and one study only looked at MI patients receiving medical therapy (Sharma et al), which is not reflective of current practice. ${ }^{13,14}$ Control group intervention also varied between studies ranging from purely pharmacological intervention to comprehensive exercise-based programmes. There were high numbers screened out in the studies, and dropout rates varied from $40 \%$ to almost nothing (Sharma et al). This does make drawing definitive conclusions difficult. We discuss the evidence from the studies on yoga's impact on cardiovascular risk, cardiac function, MACE and psychological health.

\section{Effect on Cardiovascular Risk Factors}

The RCTs looked at a variety of cardiovascular risk factors to see if the addition of yoga improved physiological and biochemical parameters.

Raghuram et al showed a statistically significant difference in reduction in BMI (reduction of $7.32 \%$ vs $1.22 \% ; p=0.001$ ) in patients who underwent a yoga-based programme compared to standard exercise-based

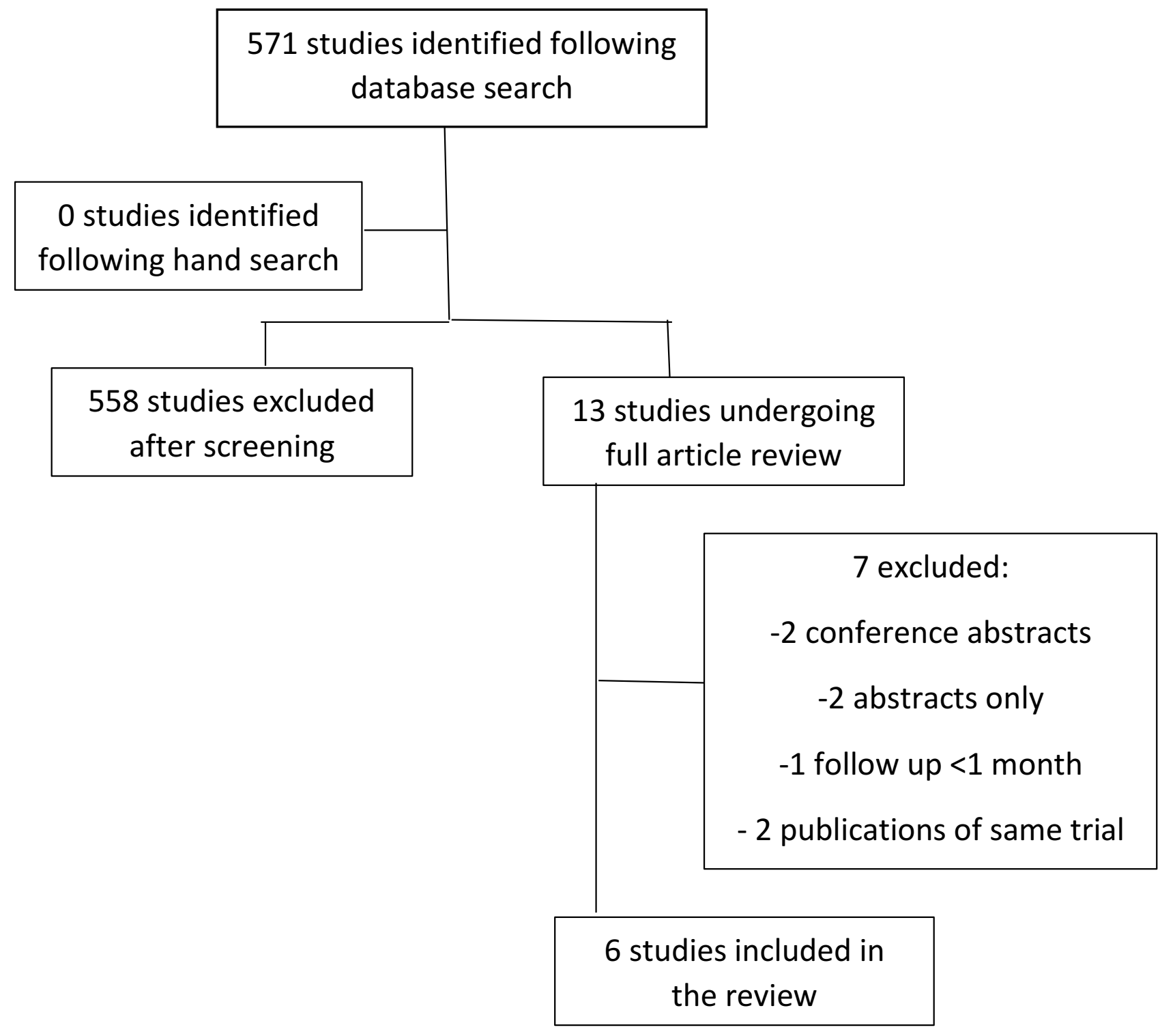

Figure I PRISMA flowchart of literature search. 


\begin{tabular}{|c|c|c|c|c|}
\hline $\begin{array}{l}\frac{y}{4} \\
\frac{\vec{y}}{x} \\
\ddot{x}\end{array}$ & 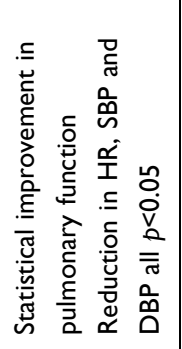 & 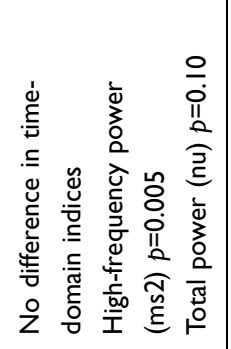 & 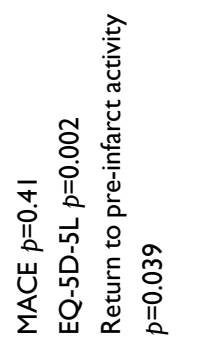 & 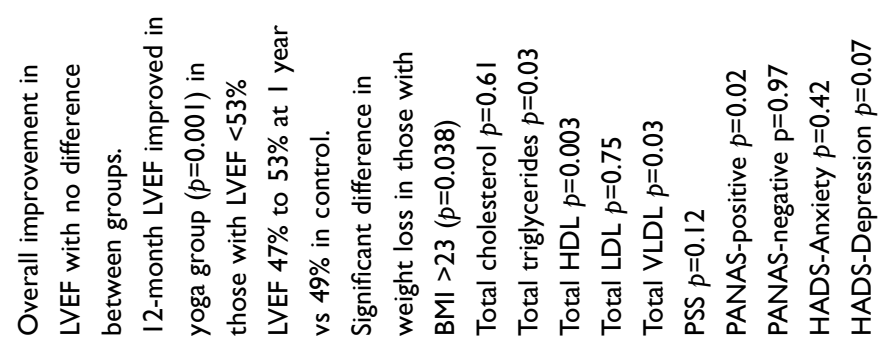 \\
\hline 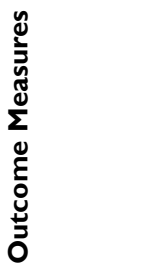 & 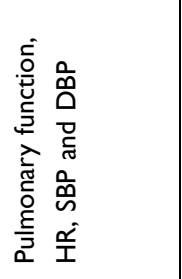 & 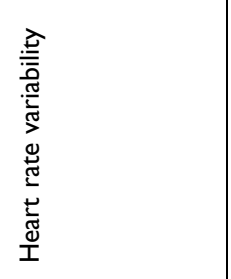 & 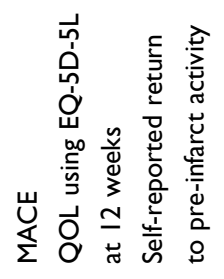 & 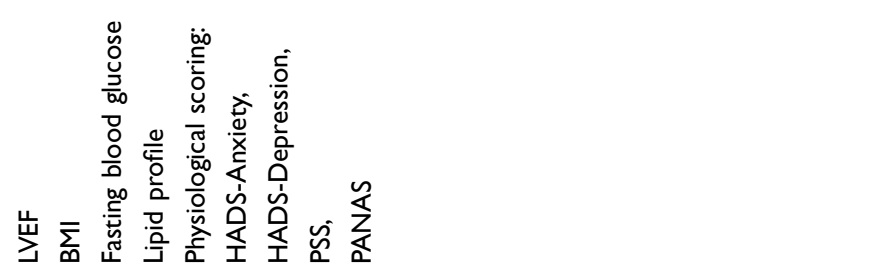 \\
\hline 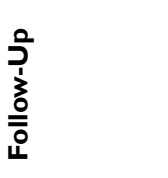 & & 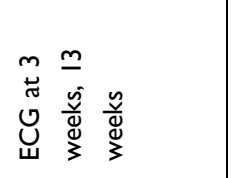 & 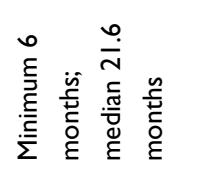 & 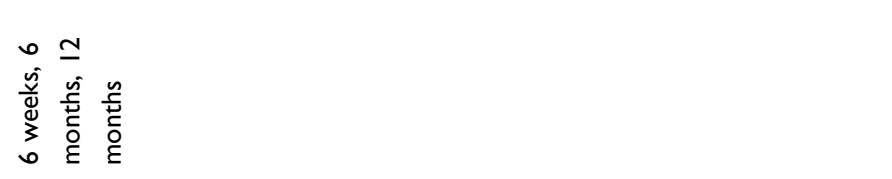 \\
\hline 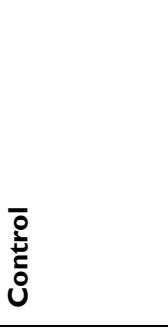 & 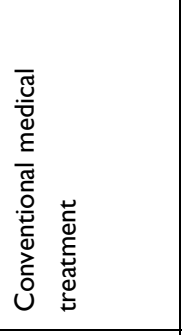 & 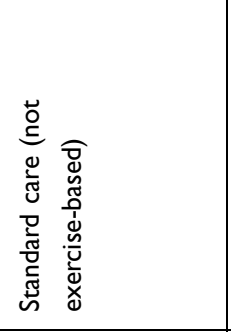 & 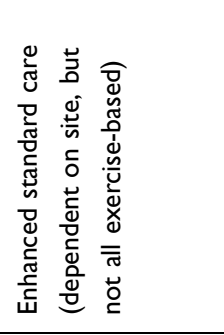 & 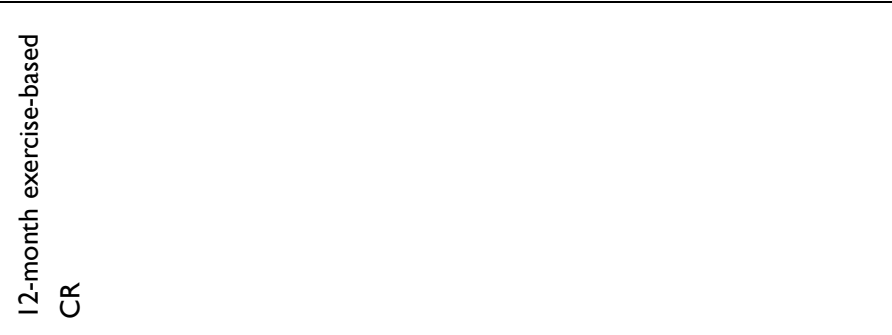 \\
\hline 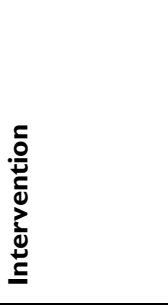 & 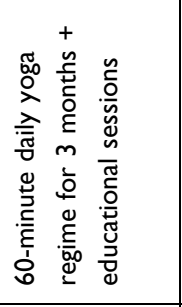 & 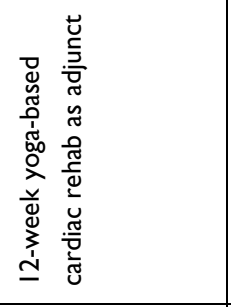 & 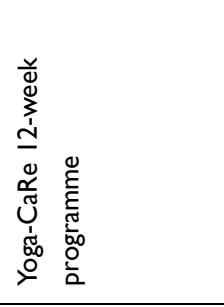 & 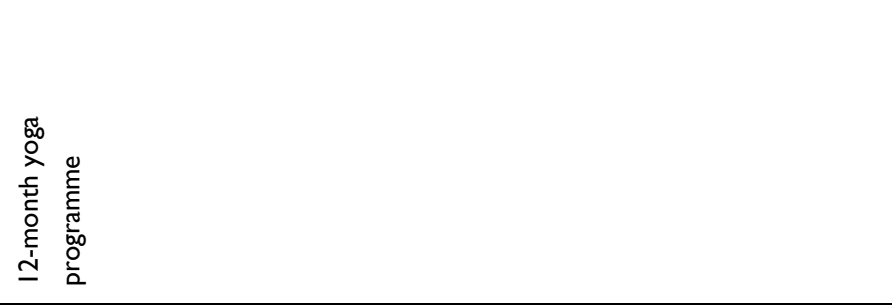 \\
\hline 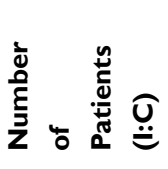 & 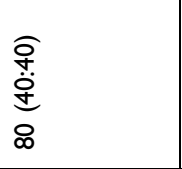 & 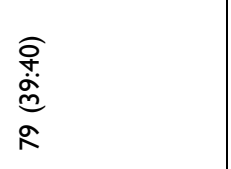 & 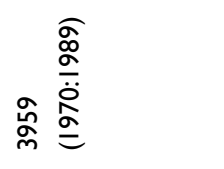 & 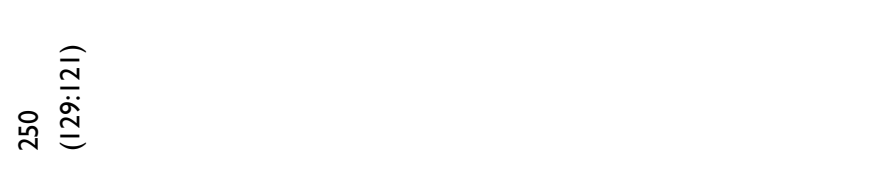 \\
\hline 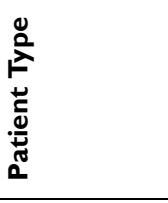 & $\begin{array}{l}0 \\
\text { U } \\
\frac{0}{0} \\
\text { 苟 } \\
\end{array}$ & $\begin{array}{l}\bar{\Sigma} \\
\dot{y} \\
\tilde{u}\end{array}$ & $\widetilde{U}$ & U⿺ \\
\hline دَّ & 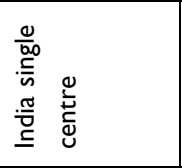 & 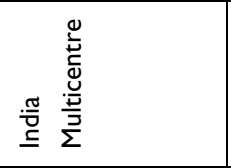 & 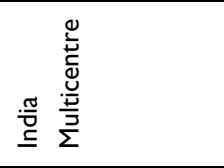 & 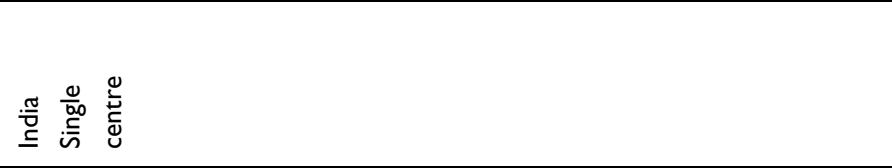 \\
\hline 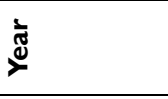 & $\stackrel{n}{\circ}$ & $\frac{a}{2}$ & ญั & $\frac{t}{i}$ \\
\hline 哣 & 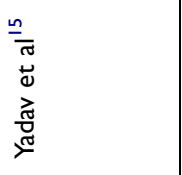 & 莺 & 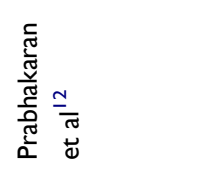 & 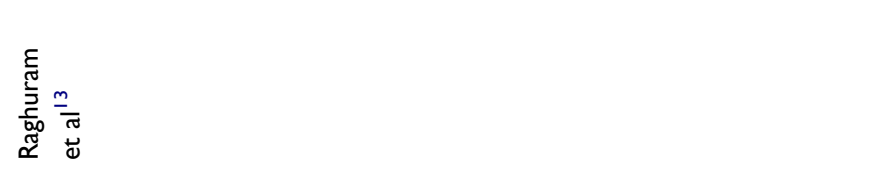 \\
\hline
\end{tabular}




\begin{tabular}{|c|c|}
\hline 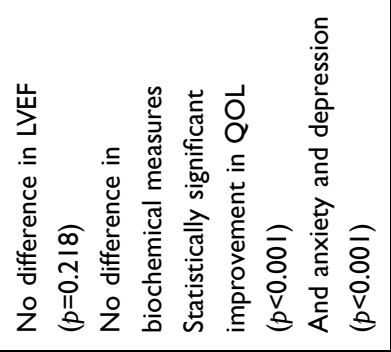 & 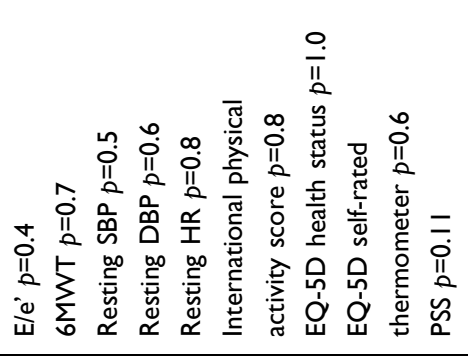 \\
\hline 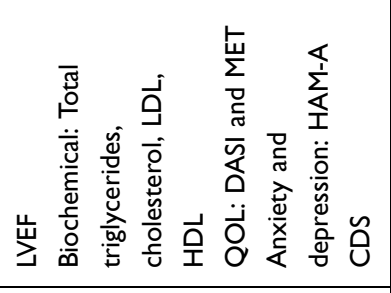 & 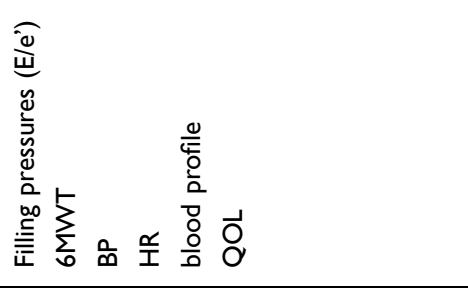 \\
\hline 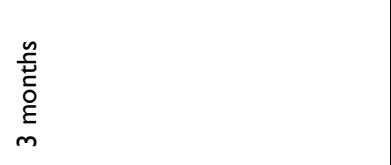 & 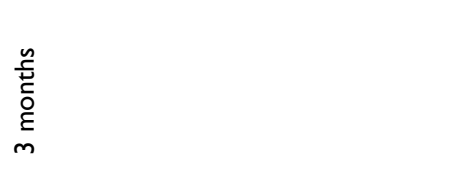 \\
\hline 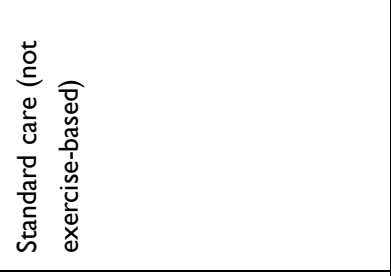 & 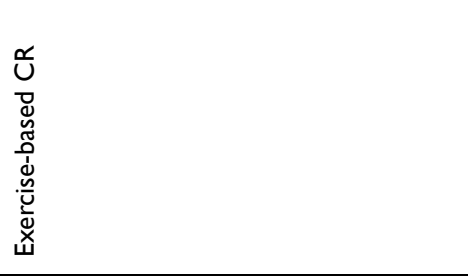 \\
\hline 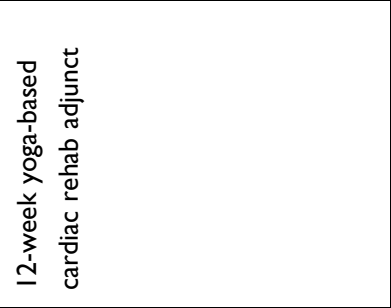 & 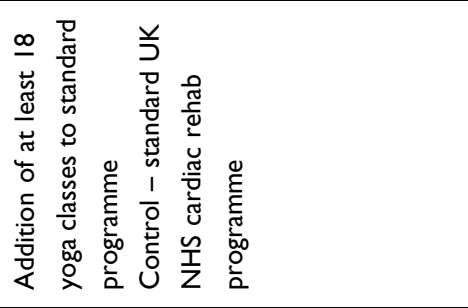 \\
\hline $\begin{array}{l}\widehat{N} \\
\stackrel{\tilde{m}}{\tilde{m}} \\
\stackrel{0}{\circ}\end{array}$ & 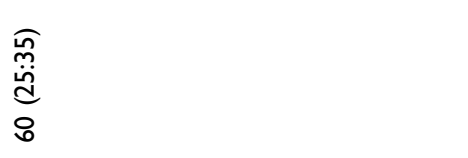 \\
\hline 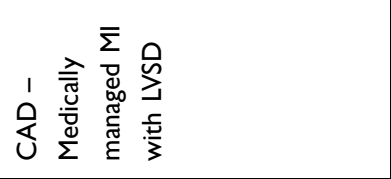 & 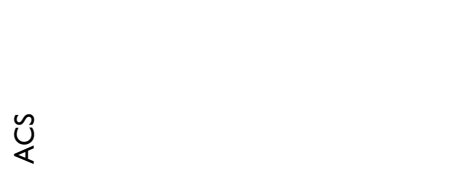 \\
\hline 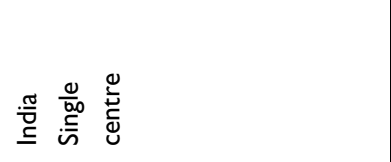 & 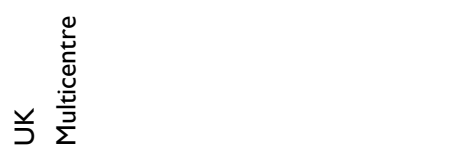 \\
\hline ర్సి & $\frac{a}{i}$ \\
\hline 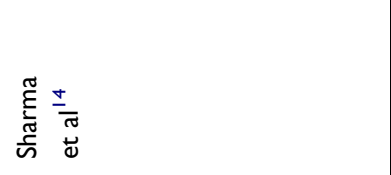 & $\begin{array}{l}\frac{0}{\bar{\pi}} \\
\stackrel{0}{0} \\
\underline{\underline{\underline{\underline{F}}}}\end{array}$ \\
\hline
\end{tabular}


rehabilitation. ${ }^{13}$ Yadav showed a statistically significant reduction in heart rate and both systolic and diastolic blood pressure when compared to solely pharmacological treatment. $^{15}$ The only non-Indian based study was carried out in the UK by Tillin et al, who found no significant difference in the physiological cardiovascular risk factors they analysed: ambulatory, resting and exertional blood pressure, resting heart rate, BMI, waist:hip ratio, percentage body fat mass and peak $\mathrm{VO}_{2} \cdot{ }^{16}$ At three months the ambulatory blood pressure in the intervention group went from an average day ambulatory systolic pressure of $115 \mathrm{mmHg}$ to $113 \mathrm{mmHg}$ and in the control group $113 \mathrm{mmHg}$ to $112 \mathrm{mmHg}(p=0.5)$. Meta-analysis of weight and systolic blood pressure favours yoga (Figures 2 and 3 ).

Three studies looked at biochemical factors associated with increased cardiovascular risk: blood glucose levels and lipid profile. Raghuram et al saw a non-statistically significant trend towards better glycemic control in the yoga group. There was a statistically significant change from baseline for those with poor control at 1 year $(p=0.008$ in baseline fasting blood glucose $>100 \mathrm{mg} / \mathrm{dL}$ and $p=0.003$ in baseline $>200 \mathrm{mg} / \mathrm{dL}$ ); however, it was not statistically significant in comparison to the control group ( $p=0.41$ and 0.032 , respectively). Tillin et al similarly did not find any statistically significant difference in blood glucose levels $(0.05 \mathrm{mmol} / \mathrm{L} ; \mathrm{CI}-0.23$ to $0.33 ; p=0.7)$. Meta-analysis again shows no statistically significant difference (Figure 4).

Raghuram et al, Tillin et al and Sharma et al looked at lipid profiles in the participants. Tillin et al and Sharma et al both found no statistically significant difference in triglycerides, total cholesterol, HDL and LDL levels at 3 months. Raghuram et al looked at the difference at baseline and following 1 year which showed a statistically significant reduction in total triglycerides $(p=0.03)$, total HDL $(p=0.003)$ and total VLDL $(p=0.03)$. No significant difference was seen in total cholesterol or total LDL between groups, though in patients with a high baseline LDL $(\geq 100 \mathrm{mg} / \mathrm{dL})$ there was significant reduction in the yoga group compared to control. Tillin et al and Sharma et al did not carry out subgroup analysis. Meta-analysis of the levels most important in cardiovascular disease risk

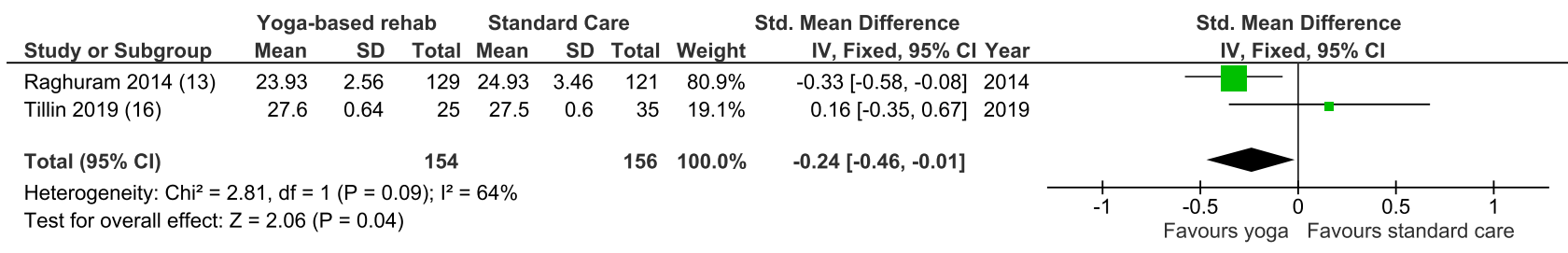

Figure 2 Meta-analysis of body mass index.

Abbreviations: SD, standard deviation; $\mathrm{Cl}$, confidence interval; IV, information value; $\mathrm{df}$, degree of freedom.

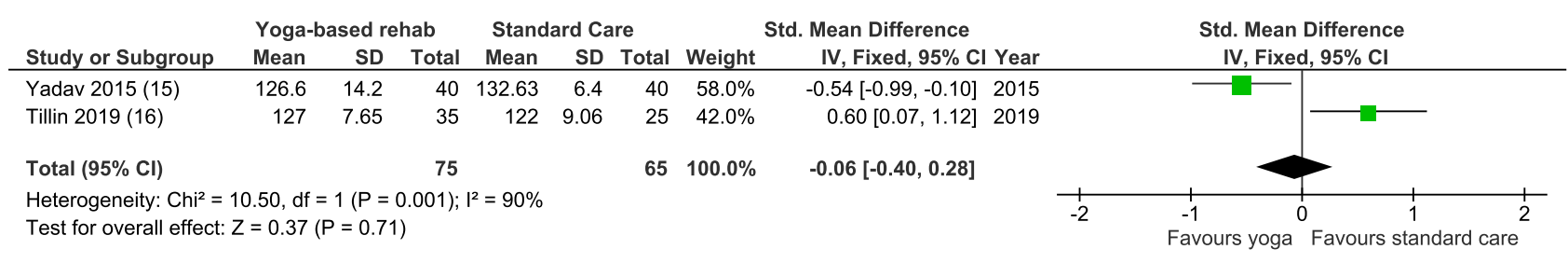

Figure 3 Meta-analysis of systolic blood pressure.

Abbreviations: SD, standard deviation; Cl, confidence interval; IV, information value; df, degree of freedom.

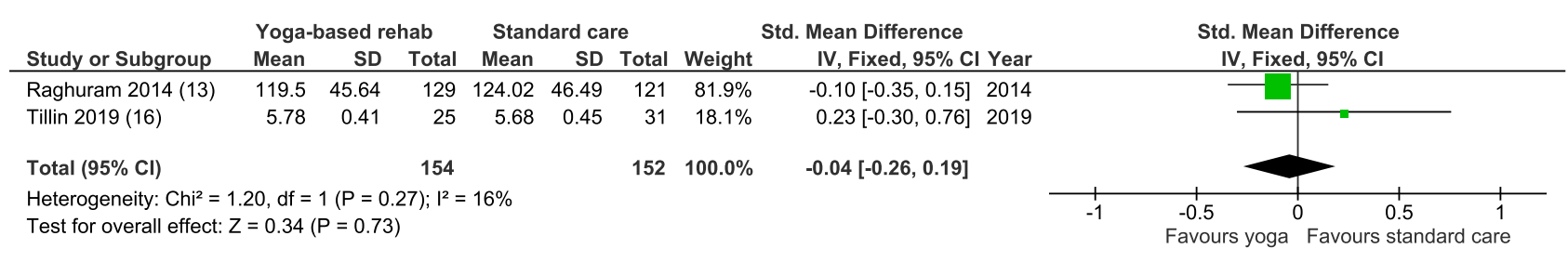

Figure 4 Meta-analysis of blood glucose levels.

Abbreviations: SD, standard deviation; Cl, confidence interval; IV, information value; df, degree of freedom. 


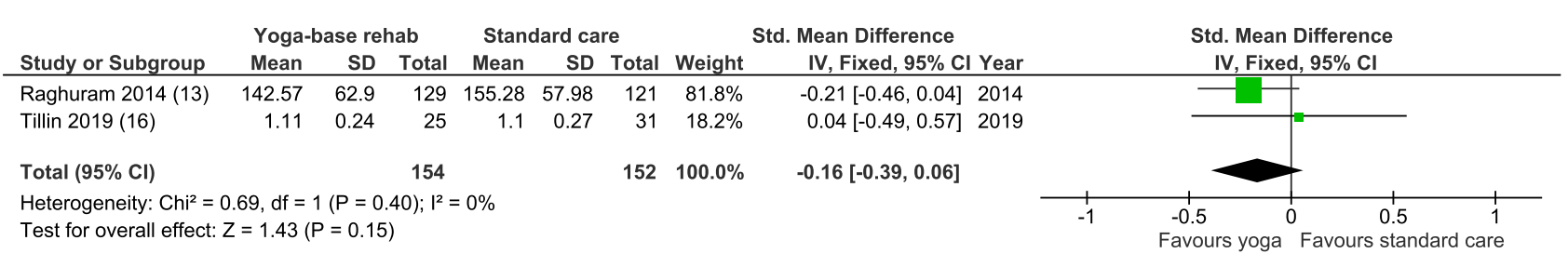

Figure 5 Meta-analysis of blood triglyceride levels.

Abbreviations: SD, standard deviation; $\mathrm{Cl}$, confidence interval; IV, information value; df, degree of freedom.

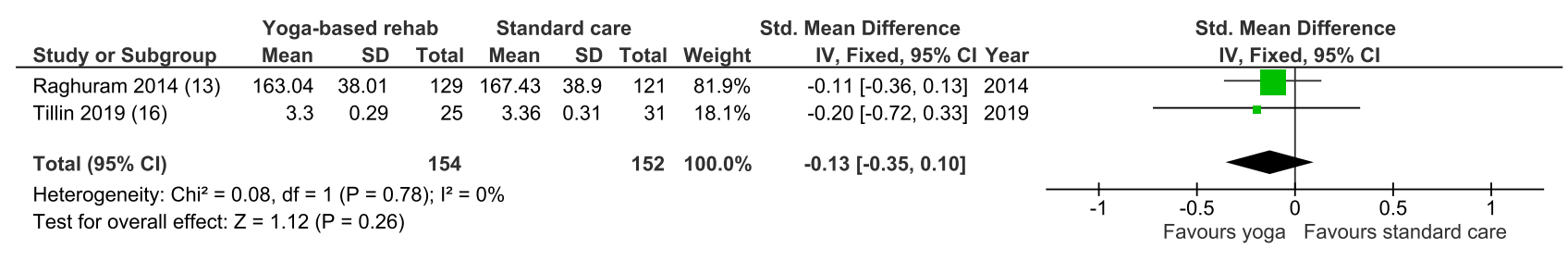

Figure 6 Meta-analysis of blood total cholesterol levels.

Abbreviations: SD, standard deviation; $\mathrm{Cl}$, confidence interval; IV, information value; df, degree of freedom.

\begin{tabular}{|c|c|c|c|c|c|c|c|c|c|c|c|c|}
\hline \multirow[b]{2}{*}{ Study or Subgroup } & \multicolumn{3}{|c|}{ Yoga-based rehab } & \multicolumn{3}{|c|}{ Standard care } & \multicolumn{3}{|c|}{ Std. Mean Difference } & \multirow{2}{*}{\multicolumn{3}{|c|}{$\begin{array}{l}\text { Std. Mean Difference } \\
\text { IV, Fixed, } 95 \% \mathrm{Cl}\end{array}$}} \\
\hline & Mean & SD & Total & Mean & SD & Total & Weight & IV, Fixed, $95 \% \mathrm{Cl}$ & Year & & & \\
\hline Raghuram 2014 (13) & 96.61 & 29.51 & 129 & 98.77 & 33.53 & 121 & $81.9 \%$ & $-0.07[-0.32,0.18]$ & 2014 & & & \\
\hline Tillin 2019 (16) & 1.76 & 0.27 & 25 & 1.81 & 0.3 & 31 & $18.1 \%$ & $-0.17[-0.70,0.36]$ & 2019 & & & \\
\hline Total $(95 \% \mathrm{Cl})$ & & & 154 & & & 152 & $100.0 \%$ & $-0.09[-0.31,0.14]$ & & & & \\
\hline \multicolumn{13}{|c|}{ 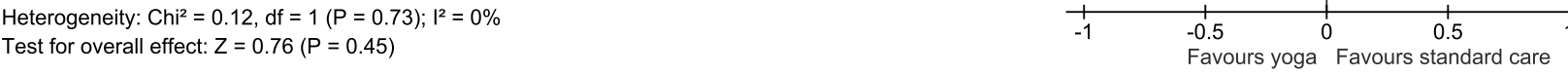 } \\
\hline
\end{tabular}

Figure 7 Meta-analysis of blood LDL levels.

Abbreviations: SD, standard deviation; $\mathrm{Cl}$, confidence interval; IV, information value; df, degree of freedom.

management showed a non-statistically significant trend favouring the yoga group (Figures 5-7). The baseline mean values for triglyceride, total cholesterol and fasting sugar level were higher in the Indian cohort studied in Raghuram et al than the UK cohort studied in Tillin et al.

\section{Improvement in Cardiac Function}

Changes in cardiac function have been looked at using clinical patient-reported measures and measurements of left ventricular ejection fraction (LVEF) and diastolic function on echocardiogram.

Clinical measures of improvement in cardiac function were looked at in three studies. Sharma et al used the Duke Activity Status Index (DASI) and derived metabolic equivalents from these self-administered functional scores to determine patient-perceived improvement in cardiac function and found those who underwent the yoga-based programme had significantly better scores at 3 months than a control group $(p<0.001)$. Prabhakaran et al also found a modest improvement in self-reported return to pre-infarct activity (88.3 vs $87.0 ; p=0.039)$ in the Yoga-CaRe group.
However, a similar questionnaire administered by Tillin et al (The International Physical Activity Questionnaire) showed no subjective improvement in perceived cardiac function between groups $(p=0.8)$.

Christa et al published data in 2019 which compared heart rate variability following acute MI in patients who underwent their 12-week yoga programme compared to standard care which did not include an exercise-based rehabilitation regime. ${ }^{17}$ They found that in patients undergoing optimal medical therapy with standard education advice, the addition of yoga interventions improved parasympathetic activity and overall cardiac autonomic tone.

Two studies looked at LVEF after yoga-based rehabilitation. Raghuram et al found that improvement in LV systolic function was significant after CABG regardless of exercise regime with no overall difference between groups. However, in patients with reduced systolic function at baseline (defined as $<53 \%$ ) there was a more significant improvement in those who underwent yoga rehab at 1 year. In the yoga group mean LV ejection fraction went from $47 \%$ to $53 \%$, while in the control group it only 


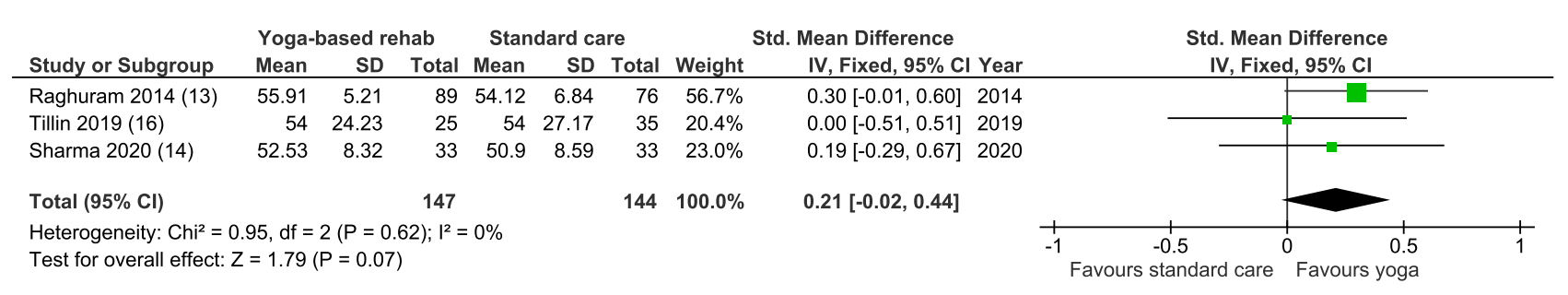

Figure 8 Meta-analysis of left ventricular ejection fraction.

Abbreviations: SD, standard deviation; $\mathrm{Cl}$, confidence interval; IV, information value; df, degree of freedom.

improved to $49 \%$ ( $p=0.02$ ). This study excluded patients with $\mathrm{EF}<30 \%$ so it is not possible to comment if those with more severe LV dysfunction would have further benefit from a low aerobic exercise programme. Sharma et al did not find a difference in LV function between the groups (this may be due to the follow-up being limited to 3 months), and the divergence was only seen after 6 months in the study by Raghuram et al. Meta-analysis did not show a statistically significant difference (Figure 8). Tillin looked at left ventricular diastolic function which was found to improve in both groups with no statistically significant difference seen in the yoga group $(p=0.04)$.

\section{Reduction in MACE}

Prabhakaran et al is the only randomized controlled trial that has looked at the reduction in MACE after yoga-based intervention. The Yoga-CaRe programme, carried out in multiple centres in India, included personalized yoga exercises, breathing control, meditation and relaxation exercises delivered by qualified yoga teachers aided by instruction booklets and training videos, which was followed by discussion of lifestyle and psychosocial concerns. The enhanced standard care programme included 3 sessions of educational advice but did not routinely offer an exercise programme.

They looked at occurrence of major adverse cardiovascular event (MACE) during follow-up, which was a composite of any cause death, non-fatal MI, non-fatal CVA or emergency cardiovascular hospitalization. The study was initially powered to detect a $20 \%$ reduction in MACE; however, it required a change to MACE criteria to include emergency cardiovascular hospitalization mid-trial to achieve higher event rates, but remained underpowered for the composite endpoint.

Between August 2014 to March 2018, they screened 6737 and enrolled 3959, and patients were well matched at baseline. They underwent median follow-up duration of
21.6 months, with MACE occurring in $6.7 \%$ of YogaCaRe and $7.4 \%$ of standard enhanced care, with a hazard ratio of 0.9 (95\% CI 0.71-1.15).

No statistically significant difference was seen in total MACE; separate MACE events were analysed, and a reduction in cardiovascular hospitalization was seen in the Yoga-CaRe group, but this was underpowered. Patient subgroups whose hazard ratio for MACE statistically favoured Yoga-CaRe were those with previous coronary artery disease $(0.49 ; 95 \%$ CI $0.29-0.81)$ and who did not have diabetes $(0.65 ; 95 \%$ CI $0.47-0.91)$.

\section{Improvement in Psychological Health}

Many studies have reported the benefits of yoga on psychological health by itself and within cardiovascular conditions. ${ }^{11,18}$ Four of the RCTs looked at patientreported scoring systems for psychological health. Sharma et al reported a significant reduction in scoring for the Cardiac Depression Scale (CDS) and Hamilton Anxiety Rating Scale (HAM-A), both with $p<0.001$. Prabhakhan et al, likewise, found their OQL questionnaire (EQ-5D-5L) results in favour of the yoga group at 12 weeks with 77 points versus 75.7, and base-adjusted mean in favour of Yoga-CaRe with 1.50 (95\% CI $0.53-$ $2.48 ; p=0.002$ ). Raghuram et al found an improvement in self-reported markers for depression, anxiety and stress in the yoga group; however, this was not statistically significantly different when compared to the control group. In their study the only area in which yoga was statistically significantly better than control was the increase in positive affectivity of the intervention on the rest of the patient's life (PANAS $p=0.02$ ). Tillin et al, however, did not find any difference between groups in their standardized QOL questionnaire (EQ-5D-3L) and perceived stress score scale. Meta-analysis of perceived stress score carried out in the studies by Raghuram et al and Tillin et al was found to favour yoga (Figure 9). 


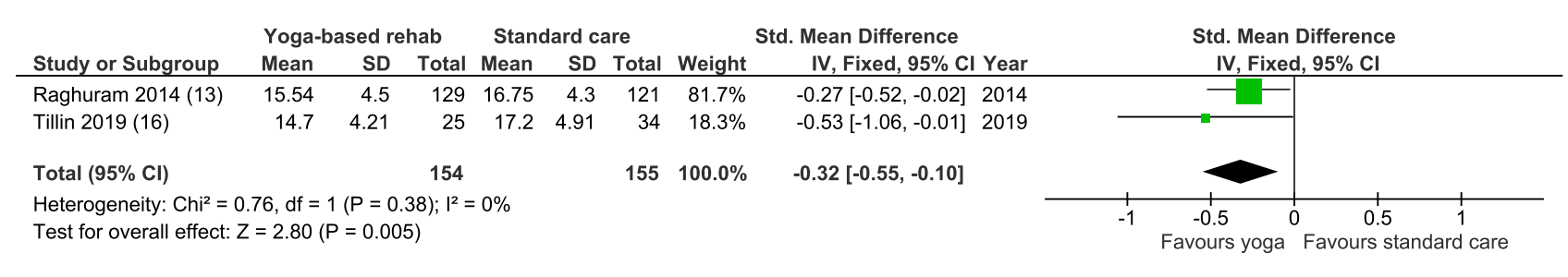

Figure 9 Meta-analysis of patient-perceived stress levels.

Abbreviations: SD, standard deviation; $\mathrm{Cl}$, confidence interval; IV, information value; df, degree of freedom.

\section{Improvement in Health Behaviours}

Prabhakhan et al's was the only study to document health behaviours between groups and found no difference between the high rates of medication adherence and tobacco cessation. This probably reflects the effects of the educational sessions in both programmes and the patient selection cohort; patients unlikely to complete the rehabilitation programme were excluded from the study. No difference in adherence to the exercise programme was seen based on age or sex of participant, once enrolled in the study.

\section{Adverse Events}

There was no increase in adverse or safety events in the yoga arms noted in any of the studies discussed.

\section{Discussion}

Yoga-based cardiac rehabilitation involves less aerobic activity than conventional exercise-based rehabilitation programmes in Europe. However, yoga-rehab still maintains the fundamental principles of cardiac rehabilitation: a structured exercise programme, which improves functional capacity in a safe environment with psychosocial support and promotion of health behaviours to reduce cardiovascular disease. In previous reports yoga has been found to improve cardiovascular health by reducing risk factors such as hypertension, heart rate variability, abdominal obesity, insulin resistance and hyperlipidemia. ${ }^{18}$ In comparison to other lifestyle interventions, yoga was found to have the largest reduction on 10-year cardiovascular risk $(16.7 \%) .^{19}$

On review of the 6 RCTs looking at yoga-based cardiac rehabilitation in coronary artery disease, there is not resounding consensus on the benefits of yoga, with the limitation of the current evidence, as documented, hampering interpretation.

One study found a reduction in patient weight, but another study looking at multiple cardiac risk factors found no significant difference between weight or any other parameter. This could be due to the small patient numbers in that study as meta-analysis does favour yoga. No statistically significant difference was seen between biochemical risks factor in studies with 3-month followup, but at 1 year Raghuram et al found reduction in total triglycerides, HDL and VLDL. Meta-analysis did favour yoga for reduction of triglycerides, total cholesterol and LDL, though without statistical significance and differing baselines and patient cohort. Most of the studies, apart from Tillin et al, reported a subjective improvement in cardiac function. An objective improvement on echocardiogram was only seen at 1 year and limited to those with reduced function at baseline. The improvement reported at 1 year was a $6 \%$ increase in ejection fraction. Ejection fraction on echocardiography is not exact and cannot be said to be reproducible, with many accreditation services now suggesting ejection fraction be given as a range. In the one study that looked at MACE there was no statistically significant improvement with yoga, and unfortunately it was unpowered for the primary outcome and subgroup analysis. Of the studies which looked at psychological health, $50 \%$ reported an improvement in patients in the yoga groups, and meta-analysis showed that the inclusion of yoga improved perceived stress scores.

There is a small number of studies with differing findings, which we think in this case reflects the heterogeneous populations studied. There is little agreement between inclusion criteria in the studies: some looked at patients after elective $\mathrm{CABG}$, some after acute MI; some included only those with LV systolic dysfunction, and others those with normal function; some studies were predominantly on males; others looked at different ethnicities with different baseline characteristics; in some, follow-up was 3 months, and in others 1 year. Often divergence in outcome was seen later in the 1-year studies, so the 3-month follow-up period is likely too short. Furthermore, most of the studies are small, and this makes drawing definite conclusions difficult. The largest study was that by Prabhakhan et al, having almost 4000 patients. However, this still was 
underpowered, and requiring the inclusion of further MACE events mid-way through the trial, which reflects how difficult it is to recruit sufficient patients to find statistical significance.

Multiple different outcomes have been looked at in these studies, but without some degree of standardized repetition it is difficult to determine the validity of results. The majority of studies were carried out in India, which makes it difficult to generalize the findings to other populations; this is reflected in the differing baseline characteristics between Raghuram et al and Tillin et al. In the only UK-based study the loss to follow-up in the yoga group was significantly higher than in the normal cardiac rehab group $(37.5 \%$ vs $12.5 \%$, respectively). This was in a small sample size (40 initial participants in the yoga arm); it was documented in the study as an unwillingness to continue with the yoga classes. From the studies there does not appear to have been increased participation in the underrepresented subgroups at conventional rehab, namely women and the elderly. Indeed, Prabhakhan et al documented a reluctance to recruit the women and the elderly, and in all studies there was a male predominance.

Previous research into barriers of cardiac rehabilitation in low- and middle-income countries reported patient, physician and systemic factors including lack of resources and affordability, and lack of patient and physician engagement in rehabilitation programmes. $^{20}$ The costs associated with a yoga-based programme are significantly less than conventional cardiac rehabilitation due to the reduced equipment and locus of classes as well as being more accessible in continents such as Asia where the practice is already widespread.

\section{Conclusion}

Overall, the studies support that the addition of yoga to rehabilitation programmes improves the subjective feeling of cardiac health and quality of life. There was also a trend towards an improvement in left ventricular systolic function in those who started with an impaired function. Improvement in cardiac risk factors, MACE and psychological health in this cohort has still to be proven, but was not inferior to standard or enhanced care, and the benefits became more pronounced at longer follow-up.

Patients who underwent yoga-based cardiac rehabilitation showed improvement from baseline in multiple domains, and while not proven to be superior, yogabased cardiac rehabilitation might be a suitable alternative rehab, especially in communities where engagement is likely to be better and where patients are therefore likely to persevere beyond the initial programme. It could also be considered as a low-cost alternative where infrastructure and recruitment of the MDT may be challenging or where distance to facilities is a barrier to treatment.

The benefits in autonomic function may also be important in other areas of cardiac rehabilitation, most notably in the setting of heart failure. Future studies with longer follow-up and larger patient numbers would aid in accurately assessing the long-term benefit of yoga-based rehabilitation.

\section{Disclosure}

The authors report no conflicts of interest in this work.

\section{References}

1. Organisation WH. Cardiovascular Disease: Key Facts. WHO; 2021. Available from: https://www.who.int/news-room/fact-sheets/detail/ cardiovascular-diseases-(cvds). Accessed November 22, 2021.

2. BHF. Heart and Circulatory Disease Statistics 2020. Institute of Applied Health Research at the University of Birmingham; 2020.

3. Anderson L, Oldridge N, Thompson DR, et al. Exercise-based cardiac rehabilitation for coronary heart disease: cochrane systematic review and meta-analysis. $J$ Am Coll Cardiol. 2016;67(1):1-12. doi:10.1016/j.jacc.2015.10.044

4. Supervia M, Turk-Adawi K, Lopez-Jimenez F, et al. Nature of cardiac rehabilitation around the globe. EClinicalMedicine. 2019;13:4656. doi:10.1016/j.eclinm.2019.06.006

5. Thomas RJ, Balady G, Banka G, et al. 2018 ACC/AHA Clinical Performance and Quality Measures for Cardiac Rehabilitation: a Report of the American College of Cardiology/American Heart Association Task Force on Performance Measures. J Am Coll Cardiol. 2018;71(16):1814-1837. doi:10.1016/j.jacc.2018.01.004

6. Perk J, De Backer G, Gohlke H, et al. European Guidelines on cardiovascular disease prevention in clinical practice (version 2012). The Fifth Joint Task Force of the European Society of Cardiology and Other Societies on Cardiovascular Disease Prevention in Clinical Practice (constituted by representatives of nine societies and by invited experts). Eur Heart J. 2012;33 (13):1635-1701. doi:10.1093/eurheartj/ehs092

7. Ranjita R, Hankey A, Nagendra HR, Mohanty S. Yoga-based pulmonary rehabilitation for the management of dyspnea in coal miners with chronic obstructive pulmonary disease: a randomized controlled trial. J Ayurveda Integr Med. 2016;7(3):158-166. doi:10.1016/j. jaim.2015.12.001

8. Gomes-Neto M, Rodrigues ES, Silva WM, Carvalho VO. Effects of yoga in patients with chronic heart failure: a meta-analysis. Arq Bras Cardiol. 2014;103(5):433-439. doi:10.5935/abc.20140149

9. Nagarathna R, Nagendra HR, Majumdar V. A perspective on yoga as a preventive strategy for coronavirus disease 2019. Int J Yoga. 2020;13(2):89-98. doi:10.4103/ijoy.IJOY_22_20

10. Geneen LJ, Moore RA, Clarke C, Martin D, Colvin LA, Smith BH. Physical activity and exercise for chronic pain in adults: an overview of Cochrane Reviews. Cochrane Database Syst Rev. 2017;4:CD011279.

11. Cramer H, Lauche R, Langhorst J, Dobos G. Yoga for depression: a systematic review and meta-analysis. Depress Anxiety. 2013;30 (11):1068-1083. doi:10.1002/da.22166

12. Prabhakaran D, Chandrasekaran AM, Singh K, et al. Yoga-based cardiac rehabilitation after acute myocardial infarction: a randomized trial. J Am Coll Cardiol. 2020;75(13):1551-1561. doi:10.1016/j. jacc.2020.01.050 
13. Raghuram N, Parachuri VR, Swarnagowri MV, et al. Yoga based cardiac rehabilitation after coronary artery bypass surgery: one-year results on LVEF, lipid profile and psychological states-a randomized controlled study. Indian Heart J. 2014;66(5):490-502. doi:10.1016/j. ihj.2014.08.007

14. Sharma KNS, Pailoor S, Choudhary NR, Bhat P, Shrestha S. Integrated yoga practice in cardiac rehabilitation program: a randomized control trial. J Altern Complement Med. 2020;26(10):918-927. doi:10.1089/acm.2019.0250

15. Yadav RK. Effect of yoga regimen on lung functions including diffusion capacity in coronary artery disease patients. Int $J$ Yoga. 2015;8(1):68-69. doi:10.4103/0973-6131.146067

16. Tillin T, Tuson C, Sowa B, et al. Yoga and Cardiovascular Health Trial (YACHT): a UK-based randomised mechanistic study of a yoga intervention plus usual care versus usual care alone following an acute coronary event. BMJ Open. 2019;9(11):e030119. doi:10.1136/ bmjopen-2019-030119
17. Christa E, Srivastava P, Chandran DS, et al. Effect of yoga-based cardiac rehabilitation on heart rate variability: randomized controlled trial in patients Post-MI. Int J Yoga Therap. 2019;29(1):43-50. doi:10.17761/2019-00019

18. Cramer H, Lauche R, Haller H, Steckhan N, Michalsen A, Dobos G. Effects of yoga on cardiovascular disease risk factors: a systematic review and meta-analysis. Int $J$ Cardiol. 2014;173(2):170-183. doi:10.1016/j.ijcard.2014.02.017

19. Chu P, Pandya A, Salomon JA, Goldie SJ, Hunink MG. Comparative effectiveness of personalized lifestyle management strategies for cardiovascular disease risk reduction. J Am Heart Assoc. 2016;5(3): e002737. doi:10.1161/JAHA.115.002737

20. Ragupathi L, Stribling J, Yakunina Y, Fuster V, McLaughlin MA, Vedanthan R. Availability use, and barriers to cardiac rehabilitation in LMIC. Glob Heart. 2017;12(4):323-34.e10. doi:10.1016/j. gheart.2016.09.004

\section{Publish your work in this journal}

Vascular Health and Risk Management is an international, peerreviewed journal of therapeutics and risk management, focusing on concise rapid reporting of clinical studies on the processes involved in the maintenance of vascular health; the monitoring, prevention and treatment of vascular disease and its sequelae; and the involvemen of metabolic disorders, particularly diabetes. This journal is indexed on PubMed Central and MedLine. The manuscript management system is completely online and includes a very quick and fair peerreview system, which is all easy to use. Visit http://www.dovepress. com/testimonials.php to read real quotes from published authors. 